\title{
Entre éloge et blâme : polyphonie et critique du tourisme dans Lanzarote et Plateforme de Michel Houellebecq
}

Praise or Blame? Polyphony and the Critique of Tourism in Michel Houellebecq's Lanzarote and Plateforme

\section{Carole Delaitre}

\section{(2) OpenEdition}

\section{Journals}

Electronic version

URL: https://journals.openedition.org/aad/5735

DOI: $10.4000 /$ aad. 5735

ISSN: 1565-8961

\section{Publisher}

Université de Tel-Aviv

\section{Electronic reference}

Carole Delaitre, "Entre éloge et blâme : polyphonie et critique du tourisme dans Lanzarote et Plateforme de Michel Houellebecq", Argumentation et Analyse du Discours [Online], 27 | 2021, Online since 14 October 2021, connection on 16 October 2021. URL: http://journals.openedition.org/aad/5735 ; DOI: https://doi.org/10.4000/aad.5735

This text was automatically generated on 16 October 2021.

\section{(c)}

Argumentation \& analyse du discours est mis à disposition selon les termes de la licence Creative Commons Attribution - Pas d'Utilisation Commerciale - Pas de Modification 4.0 International. 


\title{
Entre éloge et blâme : polyphonie et critique du tourisme dans Lanzarote et Plateforme de Michel Houellebecq
}

\author{
Praise or Blame? Polyphony and the Critique of Tourism in Michel Houellebecq's
} Lanzarote and Plateforme

\author{
Carole Delaitre
}

1 La critique du tourisme dans les récits de voyage n'est pas nouvelle. Elle a même longtemps été l'une des composantes essentielles de ce genre littéraire. Dès l'apparition du phénomène au milieu du $19^{\mathrm{e}}$ siècle se développe un trope particulier qui consiste à opposer l'auteur-voyageur aux touristes et à distinguer la pratique du premier de celle des « hordes» (Turner et Ash 1975) touristiques dont ils moquent le comportement. Ces représentations littéraires ont contribué à propager pendant un siècle et demi une image négative et stéréotypée des touristes, mais aussi du tourisme décrit comme une forme dégradée du voyage. De nombreux historiens et sociologues ont ainsi noté leur impact sur les représentations populaires en soulignant que le touriste a depuis longtemps fait sienne cette longue tradition, allant jusqu'à développer dans certains cas une véritable haine de soi $^{1}$. Conséquence de cette représentation négative, le voyage serait, selon Nathalie Roelens, producteur de récits, mais pas le tourisme : les touristes, honteux de leur statut, ne serait pas enclins à transformer leur escapade en ouvrage littéraire (Roelens 2015 : 19).

2 Cette affirmation mérite d'être nuancée puisque certains auteurs se sont interrogés de manière approfondie sur le phénomène touristique depuis la perspective de protagonistes touristes ${ }^{2}$. Michel Houellebecq en particulier peut être qualifié d'écrivain $\mathrm{du}$ tourisme dans la mesure où tous ses ouvrages de fiction mettent en scène des touristes, et deux romans en particulier (Lanzarote, 2000 et Plateforme, 2001) ont pour sujet principal le tourisme. Houellebecq lui-même ne cache d'ailleurs pas sa fascination pour cette façon de voyager puisqu'il déclarait: «Parmi les phénomènes absolument nouveaux s'étant développés au $20^{\mathrm{e}}$ siècle, celui auquel on ne peut trouver aucun réel équivalent dans les siècles antérieurs, un des plus ambigus et des moins étudiés est sans 
nul doute le tourisme» (Novak-Lechevalier 2017: 329). Peu de critiques se sont toutefois intéressés à la question du tourisme dans l'œuvre de Houellebecq malgré l'exhaustivité, la complexité et les ambiguïtés de sa réflexion ${ }^{3}$.

Cet article vise donc à explorer ce pan quasiment inédit de l'œuvre de Houellebecq et ce en étudiant les modalités discursives au gré desquelles Lanzarote et Plateforme dénoncent la rhétorique de l'industrie touristique et ses stratégies. Cette dénonciation se fait par le biais d'une double polyphonie. D'une part une polyphonie énonciative dans Plateforme puisque le lecteur apprend dans l'excipit que le récit qu'il vient de lire est en réalité celui écrit par un Michel désabusé qui considère le tourisme comme le symbole du déclin irrémédiable de l'Occident ${ }^{4}$. Le concept d'ironie tel qu'il a été défini par Oswald Ducrot est ici pertinent : «Parler de façon ironique cela revient, pour un locuteur L, à présenter l'énonciation comme exprimant la position d'un énonciateur $\mathrm{E}$, position dont on sait par ailleurs que le locuteur L n'en prend pas la responsabilité et, bien plus, qu'il la tient pour absurde " (Ducrot 1984 : 211). Cette situation énonciative est présente dans Plateforme: la révélation finale invite en effet le lecteur à déceler l'ironie de Michel-narrateur (le locuteur L) face aux opinions de Michel-personnage (l'énonciateur E) concernant le tourisme et donc à voir dans le récit de ses tribulations une critique de l'industrie touristique ${ }^{5}$. D'autre part, une polyphonie discursive est présente dans Plateforme et Lanzarote, qui permet aux narrateurs des deux romans d'exposer les stratégies mises en place dans les discours des genres dit routiniers : les catalogues de vacances et les guides touristiques (Maingueneau $2010: 31-32$ ); de mettre en avant la manière dont ils séduisent les lecteurs, notamment par des procédés de stéréotypie, mais aussi de dénoncer leurs motivations économiques et leurs fausses promesses. C'est en étudiant les procédures de mise en texte (transtextualité; intertextualité et métatextualité) que nous allons pouvoir dégager ces mécanismes de critique dans le discours du narrateur.

\section{Les catalogues touristiques : extension du domaine du stéréotype}

4 Amateurs de voyages organisés, les narrateurs de Plateforme et Lanzarote admettent, non sans gêne, avec quelle facilité ils se laissent séduire par le discours des professionnels $\mathrm{du}$ tourisme et par l'image idyllique vendue par les catalogues. Mais ils savent aussi faire preuve de distance critique par rapport à ces textes et n'hésitent pas à exposer leurs stratégies discursives, et notamment leurs recours à des stéréotypes, ces "schème[s] collectif[s] figé[s], lié[s] à la représentation commune" (Amossy et Herschberg Pierrot $2021: 100$ ).

\subsection{Une rhétorique de séduction}

5 La polyphonie énonciative mise en place par Houellebecq permet tout d'abord de dépasser le stéréotype du touriste honteux en mettant en scène des personnages qui cherchent à comprendre pourquoi les genres routiniers exercent une telle séduction sur leurs usagers. Certes, le narrateur de Lanzarote et Michel-narrateur dans Plateforme ressentent de la honte lorsqu'ils avouent être ou avoir été les clients enthousiastes des agences de voyage, sentiment qui se traduit chez le premier par une tentative de paraître décontracté pour mieux cacher son désespoir (Houellebecq $2000: 11$ ) et chez le 
second par des insultes contre sa propre personne puisqu'il se qualifie de "connard" (Houellebecq $2001: 31$ ). Mais ils sont aussi capables d'analyser leur propre attitude afin de produire une critique fondée du comportement des touristes. Dès le début de Plateforme, Michel-narrateur insiste ainsi sur la fascination qu'il éprouvait autrefois pour les brochures des agences de voyages :

Pour le décès d'un ascendant direct, on dispose dans la fonction publique d'un congé de trois jours. J'aurais donc parfaitement pu rentrer en flânant, acheter des camemberts locaux ; mais je pris tout de suite l'autoroute pour Paris. Je passai ma dernière journée de congé dans différentes agences de voyages. J'aimais les catalogues de vacances, leur abstraction, leur manière de réduire les lieux du monde à une séquence limitée de bonheurs possibles et de tarifs [...] De retour à mon travail, j'annonçais à Marie-Jeanne que j'avais besoin de vacances (Houellebecq $2001: 19-20)$.

Le jeu polyphonique donne à voir les goûts de Michel-personnage par l'intermédiaire $\mathrm{du}$ récit de Michel-narrateur. Le début de ce passage se caractérise par un ton relativement neutre, Michel-narrateur se contentant de rapporter les faits sans émettre de jugement explicite. La second partie, qui commence à "J'aimais » et peut se lire comme une tentative d'interprétation de ce comportement passé, s'avère plus ambiguë. En utilisant l'imparfait, Michel-narrateur refuse de faire siens les goûts de Michelpersonnage et porte un regard ironique sur ses agissements passés. Cette ironie naît directement de la polyphonie énonciative (Ducrot $1984: 211$ ), puisque Michel-narrateur tient ici le rôle du locuteur L et présente les opinions de Michel-personnage (l'énonciateur E) tout en refusant de les faire siennes. Si la position de Michel-narrateur n'est pas énoncée en toutes lettres, son insistance sur le décalage entre le fait de préférer les catalogues de voyage au voyage lui-même, la publicité promotionnelle à la réalité invite à une lecture ironique qui dénonce comme absurde la passion de Michelpersonnage pour la littérature touristique. Ce moment d'autodérision permet à Michelnarrateur de se placer dans une position de supériorité puisqu'il fait preuve d'une lucidité critique sur ses agissements passés tout en invitant le lecteur à s'interroger sur son propre comportement touristique. Si ce dernier est facilement séduit, comme Michel-personnage, par le discours des agences de voyage, alors il se retrouve dans la position de l'énonciateur $\mathrm{E}$ et est encouragé à corriger son comportement. Si, à l'inverse, il n'éprouve pas cette passion, le lecteur rejoint Michel-narrateur dans une position de supériorité intellectuelle gratifiante, dénonçant ainsi avec le narrateur le ridicule du touriste, consommateur de seconde main.

7 On peut toutefois se demander si le caractère implicite de la position de Michelnarrateur ne vise pas, à l'instar de l'ironie flaubertienne, à montrer que s'il faut s'opposer aux " prêt-à-dire, prêt-à-penser, prêt-à-faire, prescrits par le discours social » (et, dans le cas présent, touristique) (Amossy et Herschberg Pierrot 2021: 31), ces derniers ont aussi un «caractère inévitable " (ibid. : 32). Même si Michel-narrateur, nous l'avons dit, qualifie Michel-personnage de "connard» alors que ce dernier s'apprête à entrer dans une agence de voyage, et d'«âme moyenne " (Houellebecq 2001: 31) lorsqu'il évoque la séduction des catalogues, il n'en essaie pas moins de comprendre les raisons de sa fascination pour les discours touristiques. La formule "J'aimais les catalogues de vacances, leur abstraction, leur manière de réduire les lieux du monde à une séquence limitée de bonheurs possibles et de tarifs " peut certes être lue de manière ironique, notamment à cause de la connotation négative du verbe « réduire » et de l'insistance sur le fait que Michel-personnage accepte que le bonheur 
soit tarifé ; mais on peut aussi y voir une mise en avant de ce qui fait l'habileté de cette littérature, à savoir sa capacité à répondre à la demande d'une clientèle désabusée, prise dans l'engrenage d'une société occidentale capitaliste qui a, selon les protagonistes de Houellebecq, tout transformé en produits de consommation. Le bonheur qu'ils cherchent n'est donc pas un sentiment de plénitude totale mais un divertissement agréable à la portée de leur budget: «j'appréciais particulièrement le système d'étoiles, pour indiquer l'intensité du bonheur qu'on était en droit d'espérer. Je n'étais pas heureux, mais j'estimais le bonheur, et je continuais à y aspirer " (ibid.: 19-20). Il n'est donc pas étonnant que lorsque le narrateur évoque l'hésitation de Michel-personnage entre deux circuits, il accole au nom du séjour son prix pour souligner que, dans la rhétorique touristique, les deux sont indissociables (ibid. : 31). S'il y a jugement de la part de Michel-narrateur, il est donc surtout dirigé contre une industrie touristique qui s'inscrit dans la logique capitaliste de la société. Tout au plus se moque-t-il de la naïveté d'un Michel-personnage qui croyait encore à une forme de bonheur possible quand Michel-narrateur considère qu'une telle aspiration est vaine.

\subsection{La bivalence des stéréotypes touristiques}

8 Si la polyphonie énonciative mise en place dans Plateforme permet à Houellebecq de s'interroger sur les causes qui peuvent pousser un touriste à se laisser séduire par le discours des agences, elle permet aussi de montrer la «bivalence» du stéréotype (Amossy et Herschberg Pierrot 2021 : 36). Comme l'ont noté les psychologues sociaux américains, « le stéréotype relève d'un processus de catégorisation et de généralisation, [qui] simplifie » (ibid. : 34 ). Toutefois, un autre courant des sciences sociales insiste sur le fait que cette schématisation et cette catégorisation sont «indispensables à la cognition»: «Nous avons besoin de rapporter ce que nous voyons à des modèles préexistants pour pouvoir comprendre le monde, faire des prévisions et régler nos conduites.» (ibid. : 35). La stratégie de l'industrie touristique est ainsi de ramener le lieu à une image familière en piochant dans les stéréotypes culturels disponibles : «Par conséquent, le lecteur ne reconnaît pas un paysage, mais un locus, un topos. En grec, comme en latin, la parole et le lieu se chevauchent. Et la parole sert à ancrer le lieu dans une esthétique dominante» (Westphal et Flabbi 2009: 24-25). Alors que Michelpersonnage s'apprête à choisir un séjour touristique, Michel-narrateur déconstruit les différents degrés de catégorisation et de stéréotypes qui lui sont offerts par les catalogues de vacances. Loin de mettre en avant un lieu, les agences attirent tout d'abord les touristes en leur proposant un type de séjour et la promesse d'une vague émotion psychologique ou d'une expérience sensorielle indéterminée: "mon rêve à moi c'est d'enchaîner à l'infini les "Circuits Passion", les "Séjours couleur" et les "Plaisirs à la carte" - pour reprendre les thèmes des trois catalogues Nouvelles Frontières" (Houellebecq 2001 : 31). S'il est difficile de savoir si ces thèmes étaient réellement ceux du catalogue Nouvelles Frontières au moment de l'écriture de Plateforme, il faut toutefois noter que les catégories "séjour ", "circuit", "à la carte» et "aventure », mentionnée une page plus loin (ibid.: 32), existent sur le site du groupe allemand TUI dont fait désormais partie Nouvelles Frontières. Une fois ce premier choix effectué, le touriste est confronté à un second niveau de catégorisation. Il s'agit, pour reprendre l'expression de Westphal, de "concilier un séjour et un stéréotype " (Westphal et Flabbi $2009: 24$ ) : «J'ai tout de suite décidé de faire un circuit, mais j’ai pas mal hésité entre "Rhum et Salsa" (...) et “Tropic Thaï" » (Houellebecq 2001 : 31-32). Pour 
la Thaïlande, le catalogue a recours à la métonymie, figure récurrente dans l'industrie touristique (Westphal et Flabbi 2009: 21), puisque le pays est identifié à une zone géographique large, les Tropiques, dont la dimension dépasse considérablement la superficie de ce pays mais qui a l'avantage d'être associée à l'idée de chaleur et d'exotisme dans l'imaginaire populaire occidentale. Cuba est quant à elle désignée par deux stéréotypes culturels - une danse née sur l'île et sa boisson-phare - connotant une certaine joie de vivre. Houellebecq pastiche ici la rhétorique des agences de voyages, puisque TUI proposait en 2020 un séjour à Cuba baptisé "Rhum, Salsa et Cigares " tandis que le voyage en Thaïlande intitulé "Touks-Touks et plages tropicales", s'il porte un nom différent de celui évoqué par le protagoniste, fait lui aussi fonctionner l'imaginaire des Tropiques tout en véhiculant sa part de lieux communs.

C'est finalement la description du séjour thaïlandais qui va convaincre Michel, non parce qu'elle donnerait à voir le pays, mais parce qu'elle satisfait ses désirs médiocres en lui offrant une dernière série de stéréotypes :

Finalement, j'ai pris la Thaïlande. Il faut reconnaître que le texte de présentation de la brochure était habile, propre à séduire les âmes moyennes : "Un circuit organisé, avec un zeste d'aventure, qui vous mènera des bambous de la rivière Kwaï à l'île de Koh Samui pour terminer à Koh Phi Phi, au large de Phuket, après une magnifique traversée de l'isthme de Kra. Un voyage "cool" sous les Tropiques" (Houellebecq $2001: 32$ ).

En faisant précéder la citation d'un commentaire ironique, Michel-narrateur invite clairement le lecteur à voir l'habileté mais aussi la platitude du discours touristique. Si la description donne enfin des précisions sur les lieux visités, ces derniers semblent surtout avoir été choisis pour le degré d'exotisme qu'ils apportent, exotisme souligné par l'allitération en [k] et la répétition d'un même phonème dans un nom de lieu, deux caractéristiques rares dans la langue française. Ils sont, comme la Thaïlande dans le nom du séjour, associés à des catégories géographiques larges ("rivière ", «île", « isthme ») permettant d'évoquer l'eau et donc la possibilité de baignades bienvenues dans un climat tropical. Les précisions données sur le type de voyage renvoient par ailleurs au désir du touriste d'allier le confort d'un séjour organisé à un minimum d'« aventure » pour pouvoir s'identifier à la figure du voyageur. Enfin, il convient de noter que si le « circuit » tel qu'il est décrit n'est en rien une boucle et comporte même un certain nombre d'incohérences géographiques - la visite à l'isthme de Kra, situé au nord de Koh Samui et de Ko Phi Phi, peut difficilement être une étape entre les deux destinations - la phrase finale, qui reprend le nom du séjour, permet cependant de donner linguistiquement l'impression d'un circuit fermé. Le but du discours touristique est donc moins, selon Michel-narrateur, de mettre en avant des lieux spécifiques que de faire naître dans l'esprit du consommateur des images plaisantes liées à des stéréotypes, tout en renvoyant le touriste à une image positive de lui-même en voyageur « cool ", qualificatif qui explique certainement l'emploi de l'anglais - Tropic dans le nom du séjour qui permet de créer de l'exotisme tout en jouant sur la popularité de l'imaginaire anglo-saxon, et notamment américain, en France. Commentaire métatextuel sur l'habileté de la description, qui en programme la lecture, ou occasion d'un pastiche satirique reposant sur une exagération du style propre à cette brochure (Maingueneau $2010: 135$ ), la description du catalogue Nouvelles frontières dans Plateforme, forte d'ironie et de stéréotypes touristiques, remet en cause son caractère prescriptif et invite le lecteur à s'en méfier et à prendre ses distances avec son caractère séduisant. 
11 Outre qu'elle propage une vision stéréotypée de la Thaïlande, la description du séjour est d'autant plus problématique pour Michel qu'elle s'avère aussi trompeuse en faisant croire au touriste qu'il est un "sujet egregius " (Westphal et Flabbi 2009:16) et qu'il va vivre une expérience unique, une " aventure ", alors qu'il ne fait que partir, comme des millions d'autres, dans des lieux envahis. À la fin du roman, Michel évoque ainsi l'ouvrage d'Alex Garland The Beach (1996), et son adaptation cinématographique par Danny Boyle (sortie en 2000, un an avant la parution de Plateforme), qui met en scène des «backpackers à la recherche d'une île vierge" : "Ils se rendaient d'abord à Koh Samui, beaucoup trop touristique; de là ils gagnaient une île proche, mais il y avait encore beaucoup trop de monde pour eux. Enfin, en soudoyant un marin, ils parvenaient à débarquer sur cette île - située dans une réserve naturelle, et donc en principe inaccessible» (Houellebecq 2001: 299). En intégrant dans la description du séjour "Tropic Thaï » des références claires à ces deux œuvres (lieux mentionnés, et notamment Koh Samui et Koh Phi Phi où fut tourné le film, usage de l'anglais, et idée d'aventure), le narrateur dénonce la propension de l'industrie touristique à mobiliser les succès populaires, notamment cinématographiques, afin d'attirer les touristes. Dans leur article "The Beach, the gaze, and film tourism», les géographes Lisa Law, Tim Bunnell et Chin-Ee Ong notent en effet que le film, tout en proposant une réflexion critique sur les façons contemporaines de voyager, entraîna aussi paradoxalement le développement d'une forme particulière de tourisme, le ciné-tourisme, pratique qui consiste à visiter les lieux où ont été tournés des films (Law, Bunnell et Ong 2007 : 150-154). L'ironie du rapprochement entre The Beach et la description du séjour «Tropic Thaï » est dès lors évident. Alors que le livre de Garland est censé illustrer, selon Michel, «la malédiction du touriste, plongé dans la quête effrénée d'endroits "non touristiques" que sa seule présence contribue à discréditer " (Houellebecq 2001 : 299-300), le fait que les agences de voyages récupèrent l'œuvre artistique pour vendre des séjours organisés confirme que cette malédiction est réelle et que l'« aventure " faussement promise par le roman comme par l'industrie touristique est désormais impossible.

\subsection{Stéréotypes discursifs et clichés photographiques : des touristes consentants}

Or les touristes chez Houellebecq contribuent à leur tour à propager ces stéréotypes offerts par les supports matériels du tourisme, d'une part en répondant positivement aux offres des agences de voyages, d'autre part en désirant que les lieux correspondent aux images publicitaires mises en avant par ces dernières, images qu'ils s'empressent à leur tour de reproduire grâce à leur appareil photographique. En voyage à Lanzarote dans les îles Canaries, le narrateur du roman éponyme ridiculise ainsi l'attitude des touristes lors de la visite du Parc de Timanfaya :

Le reste de l'excursion se déroula suivant le même schéma. [...] tous les kilomètres une esplanade avait été dégagée au bulldozer, signalée à l'avance par une pancarte représentant une chambre photographique à soufflet. Nous nous arrêtions alors ; les excursionnistes, répartis sur les quelques mètres carrés de bitume, faisaient fonctionner leurs appareils (Houellebecq $2000: 21$ ).

Le passage du «nous » à l'expression « les excursionnistes » montre que le narrateur, tout en ayant conscience de faire partie du groupe, se distingue cependant de ses compagnons lorsque ces derniers exhibent une attitude touristique typique, ici la prise 
de photos compulsive. La distance critique du narrateur se lit dans l'insistance sur l'iconographie désuète qui sert à indiquer un point de vue et apparaît comme doublement déplacée dans le cadre du tourisme moderne: d'une part parce que la «chambre photographique à soufflet », objet lourd et encombrant, pourrait difficilement être utilisée lors d'un voyage en car avec arrêts photographiques fréquents sur un " espace restreint ", et d'autre part parce que sa productivité faible ne correspond pas à la frénésie photographique décrite par le narrateur. En substituant à l'attendu «ils prenaient des photos» l'expression «ils faisaient fonctionner leur appareil », ce dernier insiste sur le fait que ce qui compte pour les touristes est en effet moins d'immortaliser le paysage que d'utiliser leur appareil photo aux endroits désignés par l'industrie touristique. Les excursionnistes sont eux-mêmes dépeints comme des automates répondant mécaniquement à l'injonction des panneaux, impression renforcée par le fait que leur action se répète, comme le précise le narrateur, tous les kilomètres.

Michel dans Plateforme se moque lui aussi de l'excitation des touristes à l'idée de prendre des photographies : «Des buffles immobiles dans la boue suivaient l'autocar du regard, exactement comme l'auraient fait des vaches. Je sentis quelques trépignements du côté des écologistes jurassiens; sans doute auraient-ils souhaité réaliser deux ou trois clichés de buffles » (Houellebecq 2001: 62). Michel ridiculise la frustration des excursionnistes en banalisant le spectacle des buffles et en animalisant les touristes dont l'attitude, et notamment les "trépignements ", rappelle celui des sujets qu'ils veulent photographier. Mais là où le narrateur de Lanzarote visait surtout à critiquer le comportement grégaire des vacanciers, Michel va plus loin en montrant que les touristes contribuent, par leur comportement, à renforcer les stéréotypes que l'industrie touristique met en place. En employant le terme de "clichés " plutôt que celui de " photographies ", Michel montre que la vraie motivation des Jurassiens est de prendre une photographie "iconique » (Domšić 2013 : 278), semblable à celle diffusée dans les agences et garante de la réussite de leur voyage puisque leur expérience correspondrait à celle vendue par les catalogues. Les touristes contribuerait donc à la «mise en cliché » du monde au cœur du discours touristique selon la logique décrite par Domšić « People's perception of particular places are indebted to place-myths, that is, conglomerates of place-images in forms of clichés and stereotypes associated with particular locations, in circulation within the society. They derive their durability, spread and impact from repetition and widespread dissemination » (ibid.).

\section{Les guides touristiques : dépendance et parti-pris}

15 Apparus dans leur forme moderne à la fin $\mathrm{du} 18^{\mathrm{e}}$ siècle, les guides touristiques imprimés ont vite acquis une place essentielle dans l'expérience du voyageur et ont contribué, selon l'historien André Rausch, à transformer le voyage en tourisme (Cohen, Toulier et Vajda 2011 : 293). Prompt à ridiculiser les touristes, la littérature de voyage s'est très vite fait l'écho de cette dépendance nouvelle en faisant de la représentation du touriste guide à la main un véritable topos littéraire ${ }^{6}$. Si cette vision stéréotypée se retrouve dans les romans de Houellebecq, ses protagonistes ne se contentent pas d'attaquer les touristes. Ils font aussi subir aux guides le même traitement qu'ils ont administré aux catalogues de voyage : en les citant ou en les parodiant, ils révèlent les mécanismes de leur rhétorique et remettent ainsi en cause leur autorité. 


\subsection{Le guide touristique : un intermédiaire opaque} Houellebecq veulent certes critiquer cette relation de dépendance, mais aussi le fait que le support discursif tend, dans les cas extrêmes, à remplacer l'expérience. Cette dépendance est d'autant plus condamnable que les protagonistes sont bien conscients de la facilité avec laquelle ils se laissent parfois eux-mêmes séduire. Alors même que le narrateur de Lanzarote critique les guides au début du roman, il fait aussi souvent sien le discours de cette littérature. Sa description des paysages de l'île est ainsi à de nombreuses reprises interrompue par des passages semblant tout droit tirés d'un guide. L'évocation des vignes de la Geria en est un bon exemple :

Le soleil se couchait lorsque nous abordâmes la Geria. C'est une étroite vallée qui fraie son chemin entre des pentes de cailloux et de graviers allant du violet sombre au noir. Au cours des siècles, les habitants de l'île ont ramassé les cailloux, édifié des murets semi-circulaires, creusé dans le gravier des excavations protégées par les murets. À l'intérieur de chaque excavation, à l'abri du vent, ils ont planté un pied de vigne. [...] L'obstination qu'avait demandé ces travaux était impressionnante (Houellebecq $2000: 36$ ).

L'explication, donnée sur un ton neutre et impartial, se distingue de la narration à l'imparfait et au passé simple par l'emploi du présent de description et du passé composé pour évoquer ce mode particulier de viticulture. Elle est marquée par un bref effacement des marques d'auctorialité qui contribue à donner l'impression que le narrateur cite directement un guide sans toutefois le signaler ou en avoir conscience. Elle est ainsi parfaitement intégrée au récit qui l'encadre, d'abord parce qu'aucun signe typographique signalant une citation n'est utilisé; d'autre part parce qu'elle suit l'arrivée du groupe à la Geria et se conclut par un jugement de valeur de la part du narrateur. Le commentaire final de ce dernier porte d'ailleurs moins sur le paysage qu'il observe que sur le contenu informatif qu'il a lu, montrant ainsi que le discours touristique est totalement assimilé au point de remplacer l'expérience elle-même. Comme le note le sociologue Bernard Lerivray : «Le tourisme est devenu langage. La chose à voir est devenue langage sur la chose à voir » (Lerivray 1975 : 149).

Dans Plateforme, Michel ne peut, quant à lui, s'empêcher d'acheter Le Routard sur la Thaïlande et d'en suivre les recommandations à la lettre lorsqu'il prépare son sac (Houellebecq 2001 : 37-38). Son opinion quant à son utilité change toutefois rapidement puisqu'il finit par jeter son exemplaire dans la poubelle d'une station-service en plein milieu du séjour (102). Par ailleurs, s'il évoque à de nombreuses reprises les informations données par les guides, il le fait presque toujours pour signaler leur caractère relatif et n'hésite pas à remettre en cause leur vision du monde. À la différence du narrateur de Lanzarote, il ne fait donc jamais sien le discours des guides. $\mathrm{Au}$ contraire, il va même jusqu'à dénoncer cette attitude chez ses compagnons de voyage. En Thaïlande, il fait ainsi le portrait satirique d'un autre touriste, René, « toujours armé de son guide Michelin » (64), qui, lors de la première escale, porte plus d'attention au livre qu'au site. Ce comportement apparait comme d'autant plus étrange que René préfère vérifier dans son guide l'exactitude des propos de la jeune guide thaïe qui les accompagne plutôt que d'écouter cette dernière parler de son pays: «René, muni de son guide Michelin, suivait au fur et à mesure, toujours prêt à rectifier tel ou tel point » (62). Désireux de critiquer la pratique touristique, Michel ridiculise cette

Argumentation et Analyse du Discours, 27 | 2021 
dépendance obsessive en comparant le touriste à un élève aux premiers stades d'apprentissage de la lecture : "il lisait en suivant les lignes avec son doigt» (64). Lorsque la dépendance se fait trop grande, comme c'est le cas pour René, le guide ne remplit plus sa fonction de compagnon de voyage. Au contraire, il agit comme un écran discursif entre le touriste et le site, comme le note plus tard Michel : "Finalement, ce que cherchent avant tout les amateurs de voyages de découverte, c'est une confirmation de ce qu'ils ont pu lire dans leurs guides » (225).

\subsection{Le parti pris des guides}

Ce qui intéresse les protagonistes de Houellebecq est toutefois moins de ridiculiser les touristes que de dénoncer le parti pris des guides en déconstruisant leur rhétorique. Censés aider le voyageur dans sa découverte du pays, les guides se veulent certes pédagogiques, mais ils sont aussi idéologiquement orientés. Ils construisent les lieux autant qu'ils les décrivent, ils les popularisent et les hiérarchisent, imposant par làmême au touriste une lecture aussi bien géographique que politique de l'espace visité (Pulido, Barraclough et Cheng 2012: 4). Si le touriste est entièrement dépendant des explications de ce support discursif, il pourra facilement être trompé par un mauvais guide qui ne lui présentera qu'une vision tronquée, voire fausse, de la réalité. Bien conscient de ce risque, Michel n'hésite pas à confronter le discours des différents guides et à nuancer leur position. Il note ainsi que tous les guides s'accordent à trouver la ville thaillandaise de Surat Thani sans intérêt : «Surat Thani - 816000 habitants - se signale selon tous les guides par son manque d'intérêt absolu. Elle constitue, et c'est tout ce qu'on peut en dire, un point de passage obligé pour le ferry de Koh Samui » (Houellebecq 2001: 85). Loin d'accepter sans réagir cette opinion, Michel note d'une part la dimension hyperbolique de leurs discours (notamment par l'emploi de l'adjectif "absolu»), mais aussi leur caractère hégémonique, souligné par le «on» généralisateur qui révèle la manière dont les guides transforment un jugement individuel en vérité absolue. Par ailleurs, il souligne que la vision du lieu offerte est partielle, voire même partiale. Certes, le Guide Michelin évoque la réalité quotidienne des habitants en mentionnant l'activité économique principale - «les industries métallurgiques ", notamment dans le « domaine des constructions métalliques » (ibid.) - mais il n'en fait pas pour autant un critère suffisant pour une visite. Simple escale vers une autre destination, la ville ne fait pas pleinement partie des circuits touristiques et ne peut donc profiter financièrement du tourisme, alors même qu'elle contribue, comme le souligne Michel, à son développement :

Or, que serions-nous sans constructions métalliques? Du minerai de fer est extrait dans des régions obscures, il est acheminé par cargo. [...] La synthèse se produit dans des villes comme Surat Thani: il en résulte des autocars, des wagons de chemin de fer, des ferry-boats. [...] Le résultat sert en partie à transporter des touristes occidentaux (85).

20 En allant au-delà du discours des guides, Michel refuse ce que Paul Laurent Assoun nomme le "contrôle de l'espace [...] et du regard" mis en place par ces derniers et remet en cause leurs "prescription [...] et interdit scopiques" (Cohen, Toulier et Vajda 2011 : 281). Comme avec les catalogues, cette collision des discours touristiques et littéraires permet la subversion des premiers, par une remise en cause de ce qui est au fondement de leur fonctionnement: l'adhésion totale de leurs lecteurs aux hiérarchisations et à la vision du monde qu'ils produisent. 
21 Pour les protagonistes de Houellebecq, le parti pris des guides est visible dans la façon dont ils hiérarchisent l'espace mais aussi dans le langage qu'ils utilisent. Pour cette raison, ils ne se contentent pas de critiquer les guides en général mais se livrent à des attaques ad hominem en mentionnant très clairement le nom de leurs cibles et en citant et parodiant leur discours pour révéler leurs préjugés. Selon Michel, chaque guide vise ainsi un lectorat particulier au point qu'il affirme pouvoir connaître la personnalité de ses compagnons de voyage à partir de leur choix de guide. Josiane est ainsi associée à la mentalité du Routard, dont elle relaie, selon Michel, « la haine, [l']élitisme vulgaire et [le] masochisme agressif » (Houellebecq $2001:$ 101) et «l'objectif »: " gâcher jusqu'à la dernière petite joie des touristes" $(54)^{7}$. Cette haine s'exprime selon lui dans «les petites phrases sarcastiques » qui parsèment le guide et s'attaquent aux touristes qui ne correspondent pas à ses standards. Précis, Michel va jusqu'à en donner des exemples : «Le plus pénible était sans doute ce ton tranchant, calme et sévère frémissant d'indignation contenue : “Ce n'est pas par pudibonderie, mais nous, Pattaya, on n'aime pas. Trop, c'est trop." Un peu plus loin, ils en rajoutaient sur les "Occidentaux gras du bide" qui se pavanaient avec des petites Thaïes ; eux, ça les faisait "carrément gerber" " (54-55). Michel mêle ici intertextualité (citations), métatextualité (commentaire du narrateur qui programme la lecture de la citation), et hypertextualité (pastiche satirique) pour dénoncer la rhétorique du guide. Si la première phrase sur la pudibonderie et l'épithète "gras du bide » proviennent directement du Routard (Thailande $2001: 173,168$ ), l'expression « carrément gerber » constitue quant à elle un pastiche satirique de son style. En remplaçant le «On a la nausée » du Routard (Thailande 2001 : 452), expression appartenant au registre courant, par deux locutions familières, il exagère l'un des traits stylistiques du guide - son " élitisme vulgaire » pour mieux dénoncer son dogmatisme et son hypocrisie ${ }^{8}$.

En effet, si le narrateur s'emploie avec une telle virulence à délégitimer le Routard, c'est qu'il trouve particulièrement problématique la philosophie d'un guide qui s'est défini depuis sa création par sa "quête d'authenticité » et par "sa nostalgie des paradis perdus $»^{9}$, qu'il oppose au tourisme de masse. Comme l'a noté André Rausch, le mot d'ordre de ce guide est de "rompre» avec «le tourisme préfabriqué » pour se confronter à la vie réelle des habitants (Cohen, Toulier et Vajda 2011 : 323). Or Michel note que vanter l'authentique et prétendre le chercher n'a plus guère de sens. Il s'insurge ainsi contre Josiane qui refuse d'assister au "spectacle de "danses traditionnelles thaïes" » qu'elle juge « un peu trop touristique ». La réaction de Michel - «Qu'est-ce qu'elle voulait dire par là ? Tout est touristique » (Houellebecq 2001 : 52) montre que, pour lui, la distinction entre voyageur et touriste et entre touristique et non touristique n'a plus aucun sens dans un monde où le tourisme est devenu la norme et où l'industrie touristique a tout transformé en spectacle. Le commentaire de Josiane naît donc moins, selon Michel, d'un désir d'authenticité que d'une volonté de se distinguer d'autres touristes qu'elle juge inférieurs: "Ce spectacle de "danses traditionnelles thaïes" était selon elle tout juste bon pour Josette et René, qu'elle qualifiait en son for intérieur de bidochons » (ibid.). En convoquant le célèbre couple mis en scène dans la bande dessinée de Binet, véritable cliché des français moyens ${ }^{10}$, Michel dénonce l'hypocrisie des touristes (et de leurs guides) qui, comme Josiane, prétendent être en quête d'une authenticité que leur comportement a contribué à faire disparaitre. Conscient de vivre dans un monde où " tout est touristique ", Michel ne prétend pas être à la recherche d'une expérience authentique. Seuls demeurent selon lui des divertissements d'une plus ou moins grande médiocrité qui ne peuvent provoquer chez 
le voyageur qu'un plaisir superficiel et éphémère. En tant qu'acteurs de l'industrie touristique, les guides contribuent selon lui à cette tendance et ne jouent la carte de l'authenticité qu'à des fins commerciales, comme le font les catalogues.

\subsection{Lanzarote : un anti-Guide du Routard ?} critique son style particulier qu'il parodie lorsqu'il commence à décrire l'île :

Une dernière carte aurait pu s'offrir à l'île, celle du tourisme culturel - dont sont friands de nombreux enseignants à la retraite, et autres seniors milieu de gamme. Sur une île espagnole on pourrait, à défaut de boîtes de nuit, s'attendre à rencontrer quelques vestiges [...]. Malheureusement, l'ensemble de ces belles choses a été détruit entre 1730 et 1732 par une succession de tremblements de terre et d'éruptions volcaniques d'une violence inouïe. Donc, pour le tourisme culturel, tintin (Houellebecq $2000: 17$ ).

Si la critique Sabine Van Wesemael a noté que ce chapitre constitue «le pastiche satirique d'un mauvais guide» (Wesemael $2005:$ 141), elle ne précise toutefois pas lequel. Or il est évident - notamment au regard de la note de bas de page qui se réjouit de l'absence de Guide du Routard sur Lanzarote - que ce dernier est directement visé. Le narrateur, reprenant deux traits caractéristiques du guide, se sert d'un registre familier et emploie de nombreuses onomatopées indiquant un jugement de valeur. «L'ensemble de ces belles choses » et «tintin » sont ainsi comparables respectivement à « encore un truc incroyable » et «bof !» (Routard - Les Canaries $2016: 136)^{11}$. Le narrateur emploie par ailleurs un certain nombre de "petites phrases sarcastiques» (Houellebecq 2001 : 54), ici sur les enseignants et les seniors, afin de mieux dénoncer la tendance du guide aux stéréotypes.

Lanzarote dépasse toutefois le simple pastiche satirique. La description que le narrateur offre de l'île, si elle reprend le style du Routard pour s'en moquer, en inverse le but. Il s'agit pour le protagoniste d'écrire un anti-Routard en présentant l'île comme une antidestination pour les amateurs de ce guide : «Privé de son Guide du Routard habituel, le touriste français ordinaire risque, il faut le reconnaitre, d'éprouver rapidement à Lanzarote tous les signes d'un solide ennui » (Houellebecq 2000 : 19). Reprenant les « valeurs » chères à ce guide, qui affirme sur son site être un « melting pot d'humanisme, d'écologie, de respect des peuples, d'autodérision, d'humour bon enfant, de quête d'authenticité, de nostalgie des paradis perdus et de clins d'œil à Tintin... ", il s'en sert pour disqualifier l'île comme destination touristique ${ }^{12}$. Puisque Le Routard est célèbre pour ses "prises de position "sympa" (écologistes, humanitaires)", le narrateur affirme que «Lanzarote peut encore moins [...] se prêter au tourisme vert » (17). Afin de dissuader les routards à la " recherche quasi frénétique d'une "authenticité" en voie de disparition évidente » (18) de venir sur l'île, il dépeint cette dernière comme un enfer touristique. Enfin, loin de présenter Lanzarote comme un " coup de cœur ", l'une des expressions favorites du Routard (Les Canaries 2016 : 18), le personnage met au contraire en avant le peu d'intérêt touristique offert par l'île en concluant le chapitre par cette constatation: « une semaine dans cette île, ça devait être supportable. Pas vraiment passionnant, mais supportable » (Houellebecq $2000: 22$ ).

Or le reste du récit (et une simple recherche sur l'île) montre que cette description de Lanzarote est mensongère. Le narrateur lui-même semble d'ailleurs se contredire puisqu'il évoque dans le même chapitre deux créations de l'architecte Cesar Manrique - 
le Parc de Timanfaya et le Jardin des Cactus - qui peuvent être rangées dans la catégorie des attractions culturelles. Il prend toutefois soin d'une part de ne pas mentionner le nom de l'architecte et d'autre part de décrire ces sites comme de médiocres attractions au lieu de mettre en avant le fait qu'elles parviennent à promouvoir l'héritage culturel et l'environnement particulier de Lanzarote tout en respectant l'intégrité de son paysage : "Le "Jardin des Cactus", quoi qu'il en soit, est peu étendu; la question de la visite, pour ce qui me concerne, aurait pu être réglée en un peu moins d'une demi-heure» (19). Se contredisant de nouveau, il évoque dans la suite du récit le statut de Réserve de biosphère naturelle attribuée à l'île par l'UNESCO, preuve qu'il est donc bien possible d'y faire du tourisme vert : «Pour aller plus loin il fallait s'acquitter d'un droit d'entrée de mille pesetas, en échange de quoi on vous remettait une petite brochure avertissant de l'entrée dans une réserve mondiale de la biosphère, et énumérant différentes interdictions" (40). Toutefois, au lieu de considérer ce statut comme un moyen de protéger l'île, il préfère y voir le signe d'une arnaque visant à exploiter des touristes crédules (ibid.).

Si l'on pourrait considérer Lanzarote comme le témoignage d'un touriste déçu par ses vacances, position qui serait celle du narrateur, mais peut-être aussi celle de Houellebecq qui a visité l'île, deux facteurs permettent d'avancer une interprétation différente. Cet anti-guide peut en effet se lire non comme une critique de l'île mais bien comme une tentative d'empêcher les touristes de visiter Lanzarote afin de préserver un site que le narrateur et Houellebecq jugent exceptionnel. Il faut en effet noter que la description que le narrateur fait de l'île, loin d'en souligner la laideur, révèle au contraire que le personnage est fasciné par son étrange beauté. Ce double discours est particulièrement visible lorsqu'il décrit le site protégé par l'UNESCO précédemment mentionné. Alors qu'il dit tout d'abord du paysage qu'il «n'avait pourtant rien de spécialement remarquable", la description qu'il en donne juste après contredit totalement ce jugement négatif : « D'un seul coup, nous aperçûmes la mer. À cet endroit situé à l'extrême Sud de l'île, elle était d'un bleu idéal. [...] En deux courbes brusques, le chemin aboutit à une crique déserte. Des roches noires encadraient une pente de sable blanc qui plongeait rapidement vers la mer » (40). Par ailleurs, Houellebecq lui-même semble avoir développé une véritable fascination pour l'île, qu'il mentionne dans des poèmes mais aussi dans deux autres romans, et notamment dans La Possibilité d'une île où Lanzarote apparaît à la fin comme le lieu possible d'une utopie. Lanzarote aurait donc bien pour but d'être un anti-guide, c'est-à-dire un récit qui prendrait le contrepied de la rhétorique traditionnelle des guides visant à vanter les attraits d'un site pour mieux $\mathrm{y}$ attirer les visiteurs. La littérature chez Houellebecq servirait alors non plus à promouvoir les voyages, comme le font les discours touristiques, mais au contraire à dénoncer ces derniers et à tenter d'enrayer la progression du tourisme dans l'espoir de préserver les sites exceptionnels.

\section{Conclusion}

Romans polyphoniques reposant sur un enchevêtrement subtil de voix et sur un jeu poussé avec différents types de transtextualité, l'interprétation des récits houellebecquiens fait souvent l'objet de débats. Cette ambiguïté interprétative est particulièrement visible lorsqu'est évoquée la question du tourisme de masse. Tout en reconnaissant leur fascination pour les discours du tourisme et l'impossibilité 
d'échapper à cette forme de voyage, les narrateurs de Lanzarote et Plateforme refusent toutefois l'injonction des guides et des brochures de tourisme qui reposent avant tout sur l'adhésion sans failles du consommateur. En exposant leurs stratégies et en révélant les mécanismes de leur rhétorique, ils remettent en cause l'autorité de discours qui contribuent selon eux à transformer le monde en clichés et s'inscrivent dans la logique capitaliste destructrice des sociétés occidentales. De cette collision entre genres touristiques et littérature naît certes une critique du tourisme de masse, mais aussi un appel à des lecteurs actifs à qui il est demandé de se méfier des stéréotypes, aussi bien culturels que littéraires, et de faire preuve de vigilance par rapport à tout discours, que celui-ci soit le fait de l'industrie touristique ou des personnages houellebecquiens.

\section{BIBLIOGRAPHY}

Amossy, Ruth \& Anne Herschberg Pierrot. 2011 [1997]. Stéréotypes et clichés. Langue, discours, société. $3^{\mathrm{e}}$ éd. (Paris : A. Colin)

Baroni, Raphaël. 2016. «Comment débusquer la voix d'un auteur dans sa fiction? Une étude de quelques provocations de Michel Houellebecq ", Arborescences : revue d'études françaises 6, 72-93

Buisson, Alain. 2001. « Houellebecq sur les routes du sexe », La Dépêche (26 août)

Domšić, Lana. 2013. « Touristic photography and the construction of place identity : Visual image of Croatia », Panaitescu, Mariana, Vladimir Marascu-Klein \& Fanel-Viorel Panaitescu (eds.).

Advances in Environment, Ecocystems and Sustainable Tourism (Wseas LLC), 277-282

Ducrot, Oswald. 1984. Le Dire et le dit. (Paris : Minuit)

Garland, Alex. 1996. The Beach. (New York : Viking)

Houellebecq, Michel. 2000. Lanzarote et autres textes (Paris : Librio)

Houellebecq, Michel. 2001. Plateforme (Paris : Flammarion)

Law, Lisa, Tim Bunnell \& Chin-Ee Ong. 2007. « The Beach, the gaze and film tourism », Tourist Studies, 141-164

Lerivray, Bernard. 1975. Guides Bleus, guides verts et lunettes roses (Paris : Éditions du Cerf)

Loti, Pierre. 1990 [1908]. La Mort de Philae (Paris : France Loisirs)

McCann, John. 2010. Michel Houellebecq. Author of our times (Bern : P. Lang)

Maingueneau, Dominique. 2010. Manuel de linguistique pour le texte littéraire. (Paris : A. Colin)

Novak-Lechevalier, Agathe (éd.). 2017. Michel Houellebecq (Paris : L'Herne)

Pulido, Laura, Laura Barraclough \& Wendy Cheng. 2012. A People's Guide to Los Angeles. (Berkeley / London / Los Angeles: Univ. of California P.)

Cohen, Évelyne, Bernard Toulier \& Joanne Vajda (éds). 2011. « Le patrimoine des guides : lectures de l'espace urbain européen », Situ 15, 292-355

Roelens, Nathalie. 2015. Éloge du dépaysement - Du voyage au tourisme (Paris : Kimé) 
Turner, Louis \& John Ash. 1975. The Golden hordes : international tourism and the pleasure periphery

(London: Constable)

Urbain, Jean-Didier. 1991. L'Idiot du voyage (Paris : Plon)

Wesemael, Sabine van. 2005. Houellebecq : le plaisir du texte (Paris : L'Harmattan)

Westphal, Bertrand \& Lorenzo Flabbi (éds). 2009. Espaces, tourismes, esthétiques. (Limoges : P. U. de Limoges)

2001. Le Routard - Thailande (Paris : Hachette)

2016. Le Routard - Les Canaries (Paris : Hachette)

\section{NOTES}

1. Jean-Didier Urbain déclare ainsi dans son ouvrage L'idiot du voyage : « Le mépris antitouristique n'est pas l'apanage des indigènes extra-occidentaux et de leurs défenseurs. On le retrouve chez le touriste lui-même. Pour une part essentielle, le mépris du touriste est d'origine interne.» (Urbain $1991: 13)$.

2. Outre Michel Houellebecq qui fait l'objet de cette étude, on peut citer Jean-Paul Sartre dans La Reine Albemarle ou le dernier touriste, Violette Leduc dans Trésors à prendre, Marie NDiaye dans Un temps de saison et Ladivine ou encore Lydie Salvayre dans Les Belles âmes.

3. Parmi les critiques qui se sont intéressés à la question du tourisme chez Houellebecq, on peut citer notamment John McCann dans Houellebecq. Author of our times (2010) ou encore Sabine van Wesemael dans Le Plaisir du texte (2005).

4. Nous n'aborderons pas ici la question complexe de la position de Houellebecq sur le phénomène touristique, dont l'exploration mériterait une étude à part entière. Il convient toutefois de noter que de nombreux critiques ont tendance à attribuer à l'auteur les propos polémiques de ses personnages. Quoique critiquant les lectures biographiques, Houellebecq contribue à cet « écrasement de la polyphonie » (Baroni 2014: 80) en prêtant à ses protagonistes certains traits de sa biographie, en leur donnant ses nom et prénom, voire en reprenant à son compte leurs propos lors d'entretiens (ibid. : 77).

5. Nous ne ferons la distinction entre Michel-narrateur et Michel-personnage que lorsque le premier met clairement à distance les énoncés du second et porte sur son comportement passé un regard ironique.

6. Pour ne citer qu'un exemple, Pierre Loti emploie trois fois l'expression «le "Baedeker" à la main » dans La Mort de Philae (Loti 1990 : 35, 140-141, 146).

7. Philippe Gloaguen, le fondateur de ce guide, s'indigna d'ailleurs publiquement contre Plateforme, accusant toutefois Houellebecq non de diffamation, mais «d'écrire des saloperies contre la dignité des femmes..." (Buisson 2001). Cette accusation quelque peu décalée peut s'expliquer par le fait que Michel s'insurge notamment contre la position du guide en matière de tourisme sexuel.

8. André Rausch souligne lui aussi que l'antiélitisme du Routard s'incarne dans un style humoristique et même parfois vulgaire (Cohen, Toulier et Vajda 2011 : 327-328).

9. Ces termes sont ceux employés sur le site du Routard, sous la rubrique «La Saga du Routard » qui retrace l'histoire du guide.

10. Deux volumes de la bande-dessinée sont d'ailleurs consacrés à la question des vacances : Les Bidochons en vacances (1981) et Les Bidochons en voyage organisé (1984).

11. Comme indiqué, il n'y avait pas de guide du Routard sur Lanzarote en 2000. Celui cité ici est l'édition de 2016. 
12. Il ne serait pas étonnant que le «tintin» employé par le narrateur pour évoquer l'impossibilité de faire du tourisme culturel sur l'île soit une allusion détournée à la dernière valeur mentionnée par le Routard, les références à la bande-dessinée Tintin.

\section{ABSTRACTS}

This article analyzes the representation of mass tourism in two novels by Michel Houellebecq Lanzarote (2000) and Plateforme (2001) - and reveals that behind the apparent fascination of the protagonists for the discourses of tourism lies an unapologetic criticism of the tourist industry. In this article, I focus on the protagonists' attacks on two types of tourist discourses (tourist guides and travel brochures) and demonstrate that while they recognize their seductive power on the consumer, they also mock their formulaic and often deceptive rhetoric by using irony, playing with stereotypes, and by quoting, parodying, and pastiching their style. Ultimately, they denounce the way in which the tourist's experience is conditioned by a number of narratives on the country and its inhabitants that act as filters between the traveler and reality, and contribute to propagate a stereotypical and/or ideological vision of the world.

Cet article analyse la représentation du tourisme de masse dans deux romans de Michel Houellebecq, Lanzarote (2000) et Plateforme (2001), et montre que sous la fascination avouée des personnages pour le tourisme se cache en fait une dénonciation sévère de l'industrie touristique, et notamment des discours du tourisme. En mettant en scène des protagonistes eux-mêmes touristes mais qui font preuve de distance critique par rapport à leur propre pratique et celle de leurs congénères, Houellebecq dévoile la séduction qu'exercent les genres routiniers (catalogues de vacances et guides touristiques) sur les touristes et dénonce leurs motivations économiques et leurs fausses promesses par le recours à l'ironie, la stéréotypie, le pastiche et la parodie. Cette polyphonie discursive permet à Houellebecq d'offrir un portrait de l'industrie touristique qui semble sans cesse hésiter entre la fascination et la critique, l'éloge et le blâme.

\section{INDEX}

Keywords: Houellebecq (Michel), polyphony, tourism, travel narrative, stereotypes

Mots-clés: Houellebecq (Michel), polyphonie, récit de voyage, tourisme, stéréotypes

\section{AUTHOR}

\section{CAROLE DELAITRE}

Trinity College, Hartford 